\title{
Inhaltsverzeichnis
}

\section{Einundsechzigster Jahrgang 1966}

\section{Aufsätze}

Eißfeldt, 0.: Erwägungen zur Pentateuchquellen-

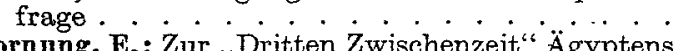
Hornung, E.: Zur ,Dritten Zwischenzeit" Ägyptens - Das thebanische Tal dor Könige.

Nagel, P.: Die Herkunft des Evangelium Veritatis in sprachlicher Sicht . . . . . . . . . . . .

Roomer, H. R.: Arabische Herrscherurkunden aus Agypten

Westendori, W.: Bemerkungen zu den Namen der Könige Djer-Athothis und Neferka. . . . . .

\section{Besprechungen}

Aalto, P.: Qutư-tu Pañcarakșā kemekü Tabun Sakiyan neretü yeke kölgen sudur, hrsg. (P. Poucha)

Aghmāti, Z.: A Digest of Commentaries on the Tractates Bābhā Kammā, Bābhā Meșī'a and Bäbhā Bhātherā of the Babylonian Talmud compiled. Ed. by J. Leveen (R. Meyer) . . .

Aistleitner, J.: Die mythologischen und kultischen Texte aus Ras Schamra, übers. 2. Aufl. (O. Eiß. feldt) . . . . . . . . . . . . . . . . .

Akurgal, E.: Die Kunst Anatoliens von Homer bis Alexander (E. P aul) . . . . . . . . . .

Alavi, B.: Geschichte und Entwicklung der modernen persischen Literatur (G. Lazard) .

Altmann, A. [Ed.]: Biblical and Other Studies (O. EiBfeldt)

Amin, E., s. Skinner, C.

Annual of Leeds University Oriental Society. Ed. J. Macdonald. II. (R. Meyer) . . . . . . . .

Archives Royales de Mari XII: Textes administratifs de la salle 5 du Palais. (2eme Partie), transcr., trad. et commentés par M. Birot (J. Aro) . .

- XIII: Textes Divers transcr., trad. et commentés par G. Dossin, J. Bottéro, M. Birot, M. L. Burke, J.-R. Kupper et A. Finet (J. Aro)

Armbruster, Ch. H. †: Dongolese Nubian (E. Dammann).

al'Ašarî, Abul-Hasan "Alî ibn Ismāeil: Die dogmatischen Lehren der Anhänger des Islam, hrog. V. H Ritter. 2. Auft. (J Fü k ) . "

'Ašiqpaśazāde, s. Kreutel, R. F'

Assyrian Dictionary of the Oriental Institute of the University of Chicago. Vol. 1 : A, I. Ed. A. L. Oppenheim (W. v. Soden)

Aufrecht, Th.: Catalogus Catalogorum. Alphabetical Register of Sanskrit Works and Authors. I.-III. (R. Hauschild).

Ausgrabungen, Neue, in Griechenland (E. Paul)

Avi-Yonah, M.: Geschichte der Juden im Zeitalter des Talmud in den Tagen von Rom und Byzanz (R. Meyer).

Babinger, F.: Spätmittelalterliche fränkische Briefschaften aus dem großherrlichen Seraj zu Stambul (B. Töpfer)

Bärwinkel, R., s. Kahlo, G.

Baidāwi, s. Beeston, A. F. L.

Bailey, H. W. [Ed.]: Indo-Scythian Studies, being Khotanese Texts. V. (O. Hansen). . . .

Baktay, E.: Die Kunst Indiens, übers. v. E. Róth (H. Mode)
Spalte

Bantu, J. K., s. Brauner, S.

Barthélemy, D.: Les devanciers d'Aquila (G. Del ling). . . . . . . . . .

Battal-raymas, A., s. Turkologie

Beeston, A. F. L.: Baidāwi's Commentary on Sūrah 12 of the Qur'an (j. Fü ck) . . . . . . . . .

Behari, B. [Ed.]: Science, Culture and $\operatorname{Man}$ (W. Ruben).

Bengtson, H., s. Handbuch der Altertumswissenschaft

Benzing, J., s. Turkologie

Betz, 0., s. Michel, O

Birot, M., s. Archives Royales de Mari XIT, XIII

Bischoff, F. E.: La forêt des pinceaux (G. Pulley blank) . . . . . . . . . . . . . . .

Blacker, C.: The Japanese Enlightenment (H)

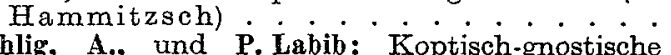

Böhlig, A., und P. Labib: Koptisch-gnostische Apokalypsen aus Codex V von Nag Hammadi im Koptischen Museum zu Alt-Kairo hrsg., übers. u. bearb. (H.-M. Schenke) . . . . . .

Boer, P. A. H. de, s. Studies on Psalms

Borst, A.: Der Turmbau von Babel, IV. (E. Hof mann) . Á Archives Royales de Mari XiIII.

Bottéro, J., s. Archives Royales de Mari XIII.
Boyd, A.: Chinese Architecture and Town Planning 1500 B. C. - A. D. 1911 (E. Bulling) . . .

Brandenstein, W., und M. Mayrhofer: Handbuch des Altpersischen (B. Schlerath) . . . . . .

Brauner, S., und J. K. Bantu: Lehrbuch des Swahili (E. Dammann)

Brecher, M.: The New States of Asia (í. $\dot{\mathrm{Ph}}$ Coolhaas).. . . . . . . . . . . . . . .

Bresciani, E. [Hrsg.]: Der Kampf um den Panzer des Inaros (G. Botti) . . . . . . . . . .

Brongers, H. A., s. Studies on Psalms

Buehberger, M., s. Lexikon für Theologie und Kirche

Burke, M. L., s. Archives Royales de Mari XIII

Burney, E. W., s. Porter, B.

Caferoğlu, A., s. Turkologie

Calmeyer, P.: Altiranische Bronzen der Sammlung Bröckelschen (E. Strommenger-Nagel) .

Carmignac, J., Cothenet, E., et H. Lignée: Les Textes de Qumran trad. et annotés. 王. (O. Eiß -

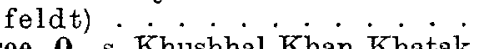

Caroe, 0., s. Khushhal Khan Khatak

Cerulli, E.: Somalia. I.-III. (H. Jensen) . . . . 194

Ch'ü T'ung-Tsu: Law and Society in Traditional China (P. Ratchnevsky) . . . . . . . . .

Clairmont, Ch. W.: The Glass Vessels (O. Eiß. feldt) . . . . . . . . . . . . . 50

Conze, E.: Buddhist Thought in India (F. Weller) 272

Cothenet, E., s. Carmignac, J.

Dalglish, E. R.: Psalm Fifty-One in the Light of Ancient Near Eastern Patternism (G. Wallis)

Dalton, 0.: The Treasure of the Oxus. 3rd ed. (W. Nagel) .............. . Dedering, S., s. as-Safadi

Delekat, L.: Katoche, Hierodulie und Adoptionsfreilassung (G. Delling) . . . . . . - . . .

Devaraja, N. K.: An Introduction to Sankara's Theory of Knowledge (L. Schmithausen) .

595 Dobson, W. A. C. H.: Early Archaic Chinese (J. Vochala). . . . . . . . . . . . . 275
Spalle 
Dossin, G., s. Archives Royales de Mari XIII

Drower, E. S.: A Pair of Nasoraean Commentaries.

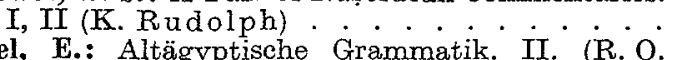
Edel, E.: Altägyptische Grammatik. II. (R. O. Faulkner)

Eißfeldt, 0.: Einleitung in das Alte Testament. 3. Auf. (S. Segert)

- s. Religionsgeschichte des Alten Orients

Emanuels, H. W., s. Teeuw, A.

Ernst, H.: Die mamlukischen Sultansurkunden des Sinai-Klosters hrsg., übers. u. erläutert (H. R. Roemer).

Finet, A., s. Archives Royales de Mari XIII

Fischel, W., s. Herford, R. T.

Fischer, F.: Die hethitische Keramik von Borazköy (M. J. Mellink)

Fischer, H.. G.: Inseriptions from the Coptite Nome (D. Müller)

Fleisch, H.: Traité de Philologie Arabe. I. $(\mathrm{K} . \dot{\mathrm{P} e}$ tráček)

Fohrer, G.: Das Buch Hiob (E. OB wald)

Fozilov, M.: Farhangi iborahoi rekhtai zaboni hozirai tojik (A phraseological dictionary of the contemporary Tadjik language). I. (J. Bečka)

Gabain, A. v., s. Turkologie

Galling, K.: Studien zur Geschichte Israels im persischen Zeitalter (O. EiBfeldt)

Geißler, F., s. Pforr, A. v.

Gemser, B. $\dagger, s$. Studies on Psalms

Gerster, G.: Nubien - Goldland am Nil (A. Her$\operatorname{mann}$ )

Gevirtz, S.: Patterns in the Early Poetry of Israel

(G. Wallis): Magic and Medical Science in Ancient Eoypt (W. Westendorf)

Grapow, H.: Wie die Alten Ägypter sich anredeten, wie sie sich grüßten und wie sie miteinander sprachen. Zweite Ausgabe (S. Herrmann).

Grohmann, A.: Arabien. (Handbuch der Altertumswissenschaft, hrsg. v. H. Bengtson, III, 4) (J. Oelsner)

- s. Philby, H.

Gurney, 0. R.: Anatolia c. $1750-1600$ B. C. (H. Klengel) . . . . . . . . . . . . .

- and P. Hulin: The Sultantepe Tablets. II. (W von Soden).

Gynz-Rekowski, G. v.: Symbole des Weiblichen in Gottesbild und Kult des Alten Testamentes (E. Oßwald)

Haenisch, E. †: Mandschu-Grammatik (W. Fuchs)

Hammerschmidt, E.: Stellung und Bedeutung des Sabbats in Äthiopien (W. Müller)

Handbuch der Altertumswissenschaft, hrsg. v. H. Bengtson, III, 4, s. Grohmann, A.

Handbueh der Orientalistik, I. Abt., Bd. V, 1: Altaistik, s. Turkologie

Hanhart, R., s. Jepsen, A.

Hansen, 0.: Mittelpersisches Lesebuch (G. La. $z$ ard)

Harders-Steinbäuser, M., s. Meisezahl, R. $\cdot$

Hardy, M. J. L.: Blood Feuds and the Payment of Blood Money in the Middle East (F. Løkke gaard)

Heissig, W.: Beiträge zur Übersetzungsgeschichte des mongolischen buddhistischen Kanons ( $P$. Poucha)

Helck, W.: Materialien zur Wirtschaftsgeschichte des Neuen Reiches. III, IV (J. v. Beckerath)

- dasselba V. (J. v. Beckerath)

Hempel, J. +: Geschichten und Geschichte im Alten Testament bis zur persischen Zeit (E. OB wald)

- s. Religionsgeschichte des Alten Orients

Hengel, M., s. Michel, O.

Henthorn, W. E.: Korea. The Mongol Invasions (P. Aalto) . . . . . . . .

Herford, R. T.: Die Pharisäer, übers. v. W. Fischel (L. Rost) . . . . . . .

Spalte

251

544

460
Hintze, F.: Die Inschriften des Löwentempels von Musawwarat es Sufra (J. Leclant) . . . . . 546

Hirschberg, W.: Schwarzafrika (H. Jungraith-

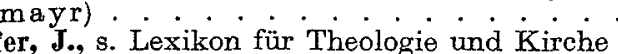

Holt, P. M., s. Lewis, B.

Hornung, E.: Das Amduat. I und II (H. Alten-

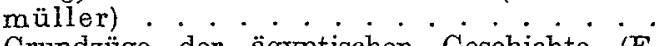

- Grundzüge der ägyptischen Geschichte (E. Blumenthal) $\dot{0} \dot{\text { Khan Khatak }}$

Howell, E., s. Khushhal Khan Khatak

Hsuieh, Ch.: Huang Hsing and the Chinese Revolution ( $\mathrm{J} . \mathrm{FaB})$

Fuffmon, H. B.: Amorite Personal Names in the Mari Texts: A Structural and Lexical Study (M. Dietrich u. O. Loretz) . . . . .

Hulin, P., s. Gurney, O. R.

[Ibn Ijās:] Die Chronik des Ibn Ijās. 2. Aufl., bearbeitet von M. Mostafa. III. (A. SchimmeI)

Jacob, E.: Ras Shamra-Ugarit et l'Ancien Testament (A. Jepsen)

Janert, K. L., s. Schubring, W.

Jarring, G.: An Eastern Turki-English Dialect Dictionary (S. Kakuk)

Jayme, G., s. Meisezahl, R. O.

Jepsen, A., und R. Hanhart: Untersuchungen zur israelitisch-jüdischen Chronologie (W.-H. Schmidt)

Jisl, L.: Mongolei. Übers. von F. u. C. Kirschner

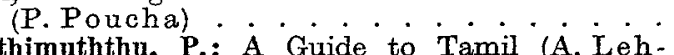
mann). . . . . . . . . . .

Kahlo, G.: Grundriß der indonesischen Sprache (C. Hooykaas) : und R. Bärwinkel: Indonesisch-deutsches Wörterbuch (C. Hooykaas)

Kees, H.: Die Hohenpriester des Amun von Karnak von Herihor bis zum Ende der Äthiopenzeit (E. Hornung)

Kenna, V. E. G.: Cretan Seals. With a Catalogue of the Minoan Gems in the Ashmolean Museum (H.-G. Buchholz).

Khushhal Khan Khatak: The Poems, transl. by E. Howell and O. Caroe (A. Schimmel) . . . Kirsehner, F. und C., s. Jisl, L.

Kitagawa, J. MI.: Gibt es ein Verstehen fremder Religionen? (O. Eißfeldt)

Klengel, H.: Geschichte Syriens im 2. Jahrtausend v. u. Z. I. (M. Dietrich u. O. Loretz).

Klingenheben, A. +: Die Sprache der Ful (H. Jung raithmayr)

Koole, J. L., s. Studies on Psalms

Koran, Der, übers. von R. Paret. 1. u. 2. Lfg (R. Blachère) . . . . . . . . . . . * .

Krahl, G.: Deutsch-Arabisches Wörterbuch, bearbeitet und herausgegeben (H. Grotzfeld).

Kraus, F. R.: Briefe aus dem British Museum (CT 43 und 44) (J. Aro)

Kreutel, R. F.: Vom Hirtenzelt zur Hohen Pforte (Die Chronik des 'Ašiqpašazäde) (G. Káldy Nagy)

Kroeber, R.: Der Prediger. Hebräisch und deutsch (O. Eißfeldt)

Külling, S. R.: Zur Datierung der ,Genesis-P.

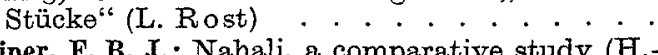

Kuiper, F. B. J.: Nahali, a comparative study (H. $J$. Pinnow)

Kupper, J.-R., s. Archives Royales de Mari XIII

Kutsch, E.: Salbung als Rechtsakt im Alten Testament und im Alten Orient (G. W allis)

Labib, P., s. Böhlig, A.

Lamotte, E.: The Spirit of Ancient Buddhism. Transl. by R. Toulmin (H. Bechert) . . . .

Leclant, J.: Les sagesses du Proche-Orient ancien (O. Eißfeldt) . $\cdot \cdot \cdot \cdot \cdot \cdot \cdot \cdot \cdot \cdot \cdot \cdot \cdot \cdot \cdot \cdot$

Leichty, E.: A Bibliography of the Cuneiform Tablets of the Kuyunjik Collection in the British Museum (W. Röllig) . . . . . . . . . palte 
Leisterer, H., s. Otzen, B.

Leveen, J., s. Aghmāti, Z.

Lewis, B.: The Middle East and the West (B. Spuler)

- and P. M. Holt [Ed.]: Historians of the Middle East (M. Fleischhammer). . . . . . .

Lexikon fuir Theologie und Kirche. Begx. von M. Buchberger. 2. Aufl., hrsg. von J. Höfer und K. Rahner. IX. (H. Bard tke)

Lienhard, S.: Tempusgebrauch und Aktionsartenbildung in der modernen Hindi (H. Berger) .

Lignée, H., s. Carmignac, J.

Lippens, P., s. Philby, H.

Lucas, H.: Lamaistische Masken (J. Schubert) .

Macdonald, J., s. Annual of Leeds Univ. Oriental Society

- s. Memar Marqah

Mahmassani, S.: Falsafat al-Tashri fi al-Islām, transl. by F. J. Ziadeh (J. Fü ck) . . . . . .

Makdisi, G.: Ibn 'Aqil et la résurgence de l'Istam traditionaliste au XIe siècle (A. Schimmel) .

Mallowan, M. E. L., s. Woolley, L.

Mansoor, M.: The Dead Sea Serolls (O. Ei $B$ feldt)

Masselman, G.: The Cradle of Colonialism (W. Markov) Coiran et la révélation Judéo. Masson, D.: Le Coran et la révélation JudéoChrétienne. I, II. (L. Rost)

Mayrhofer, M., s. Brandenstein, W.

Meisezahl, R. 0., Harders-Steinhäuser, M., und G. Jayme: Alttibetische Handschriften der Völkerkundlichen Sammlungen der Stadt Mannheim im Reiß-Museum (M. Taube). . .

Memar Marqah. The Teaching of Marqah, ed. and transl. by J. Macdonald. I und II (H.-F. Wei B)

Menges, K. H., s. Turkologie

Merkelbach, R.: Isisfeste in gxiechisch-römischer Zeit. Daten und Riten (H.-D. Altendorf)

Mesnil du Buisson: Les Tessères et les Monnaies de Palmyre (O. Ei i feldt)

[Michel, 0.:] Abraham unser Vater. Festschrift für Otto Michel zum 60. Geb., hrsg. v. O. Betz, M. Hengel, P. Schmidt (G. Ba umbach) .

Mishra, Y.: An Early History of Vaiśāli (L. Skur$\mathrm{zak}$ )

Morag, Sh.: The Vocalization Systems of Arabic, Hebrew, and Aramaic (R. Meyer). . . . . .

Moss, R. L. B., s. Porter, B.

Mostala, M., s. Ibn Ijäs

Mowinckel, S.: Erwägungen zur Pentateuchquellenfrage (O. Eißfeldt) . . . . . . . .

- Tetrateuch - Pentateuch - Hexateuch (E. $\mathrm{OBw}$ ald)

Olschak, B. C.: Religion und Kunst im alten Tibet (F. Weller)

Oppenheim, A. L., s. Assyrian Dictionary

- Studies presented to A. Leo Oppenheim (K.

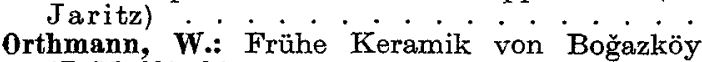
(J. Mellink) :

otten, H., s. Religionsgeschichte des Alten Orients

- und V. Souček: Das Gelübde der Königin Puduhepa an die Göttin Lelwani (K. Riemschneider).

0tto, E.: Gott und Mensch nach den ägyptischen Tempelinschriften der griechisch-römischen Zeit (E. Blumenthal).

- Aus der Sammlung des Ägyptologischen In stitutes der Universität Heidelberg (A. Her. man $n$ )

- s. Religionsgeschichte des Alten Orients

Otzen, B.: Studien über Deuterosacharja, übers. v. H. Leisterer (H.-J. Zobel) . . . . . . . .

Oudenrijn, M.-A. van den: Gamaliel. Äthiopische Texte zur Pilatusliteratur (E. Hammerschmidt)

Pandey, B. N., s. Philips, C. $\dot{H}$.

Papoulia, B.: Ursprung und Wesen der ,Knabenlese" im osmanischen Reich (J. Reychman)
Spalte

Paret, R., s. Koran, Der

Spalte

Peisker, C. H.: Hebräische Wortkunde (R. Meyer) 467

Pen-Tsi King. Ouvrage taoïste inédit du VIIe siècle (A. F. P. Hulsewé)

Pforr, A. v.: Das Buch der Beispiele der Alten Weisen. Kritisch hrsg. nach der Straßburger Handschrift. I: Text, hrsg. v. F. Geißler (B. Holbek)

Philby, H., Ryckmans, G., et P. Lippens: Expédition en Arabie. IIe Partie: Textes épigraphiques. Tome 1: Arabic Inscriptions. By A. Grohmann (W. W. Müller)

Philips, C. H., Singh, H. L., and B. N. Pandey: The Evolution of India and Palkistan 1858 to 1947 (L. Alsdorf)

Piper, A., s. Reischauer, E. O.

Ploeg, J. van der, s. Studies on Psalms

Poppe, N., s. Turkologie

Porter, B. †, Moss, R. L. B., and E. W. Burney: Topographical Bibliography of Ancient Egyptian Hieroglyphic Texts, Reliefs, and Paintings. I, 2. 2nd ed. (E. Hornung)

Porubean, S.: $S$ in in the old Testament (R. Hentschke) . . . . . . . . . . . . . . .

Pritchard, J. B.: The Bronze Age Cemetery at

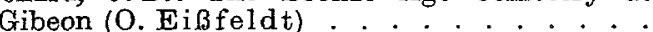

Pritsak, 0., s. Turkologie

Qureshi, I. H.: The Muslim Community of the Indo-Pakistan Subcontinent $\quad(610-1947)$ (J. Fü ck).

Rabel, L.: Khasi, a Language of Assam (H. Ber ger) . • Das fünfte Buch Mose, Deuteronomium übersetzt und erklärt (O. Eißfeldt).

Rahner, K., s. Lexikon für Theologie und Kirche

Reischauer, E. 0.: Die Reisen des Mönchs Ennin. Übers. von A. Piper (J.W. de Jong) . . . .

Religionsgeschichte des Alten Orients. Lfg. 1. Mit Beiträgen v. O. Eißfeldt, J. Hempel, H.Otten, E. Otto (K. Rudolph). . . . . . . . . .

Renou, L.: Etudes védiques et pāninéennes. Xं et XII (W. Rau). . . . . . . . . . . . .

Richardson, H. E.: Tibet and its History (J. Schu bert)

Ridderbos, N. H., s. Studies on Psalms

Ringgren, H.: Israelitische Religion (O. EiBfeldt)

Ritter, H., s. al" Aš ari

- s. aș-Şafadi

Roth, E., s. Baktay, E.

Ryckmans, G., s. Philby, H.

[aș-Ṣafadī:] Das biographische Lexikon des Șālāhnaddin Halil Ibn Aibak aș-Safadi. IV., hrsg. v. S. Dedering. I., 2. Aufl., hrsg. v. H. Ritter (M. Fleischhammer) ......... (M. Fleischhammer).

Schacht, J.: An Introduction to Islamic Law (W. M. W att). . . . . . . . . . . . . . . . .

Schafer, E. H.: The Golden Peaches of Samarkand (J. Chmielewski)

Scharbert, J.: Heilsmittler im Alten Testament und im Alten Orient (‥ OBwald). . . . . . . Sehmidt, P., s. Michel, $O$.

Sehottroff, W.: ,Gedenken" im Alten Orient und im Alten Testament (E. OBwald). . . . . . Schubring, $W$. [Hrsg.]: Indische Handschriften. I., beschrieben von K. L. Janert (F. Weller)

Shaki, M.: Moderní perská frazeologie a konverzace. (A modern Persian phrase-book) (J. Bečka) . . . . . . . . . . . . . .

Shorto, H. L. [Ed.]: Linguistic Comparison in South East Asia and the Pacific (N. M. Holmer

Sibinga, J. S.: The Old Testament Text of Justin Martyr. I. (J. Hempel $\dagger$ )..........

Siegler, K. G., s. Stock, H.

Singh, H. L., s. Philips, C. H.

Sinor, D.: Introduction à l'étude de l'Eurasie Centrale (K. H. Menges)

\section{0}


Skimner, C.: Sja'ir Perang Mengkasar (The Rhymed Chronicle of the Macassar War) by Entji' Amin, ed. and transl. (H. J. de Graaf). . . . . . . Snijders, L. A., s. Studies on Psalms

Sorokin, W.F.: Der Schaffensweg Mao-tuns (F. Gruner)

Součk, V., s. Otten, $\dot{H}$.

Spies, 0., s. Turkologie

Stein, R.-A.: Recherches sur l’épopée et le barde au Tibet ( $J$ Schubert)

Stern, S. M.: Fātimid Decrees (B. Spuler) . . . .

Sternbach, L.: Cänakya-Niti-Text-Tradition. Vol. I, Part I, 1-4 (W. Ruben) . . . . . . . . .

- Cārnakya-Rāja-Niti, Maxims on Rāja-Niti (W. Ruben) . . . . . . . . . . . . . . .

Stoek, H., und K. G. Siegler: Kalabsha (A. Her $\operatorname{mann})$. . . . . . . . . . . . . . . . . .

Street, J. C.: Khallhha Structure (H.-P. Vietze)

Studies on Psalms. By B. Gemser $\dagger$, H. A. Brongers, N. H. Ridderbos, G. J. Thierry $\dagger$, J. L. Koole, I. A. Snijders, A. S. van der Woude, J. van der Ploeg, P. A. H. de Boer (G. W allis)

Suzuki, D. T.: Zen and Japanese Culture. Rev. and enlarged 2nd ed. of Zen Buddhism and Its Influence on Japanese Culture (P. Demié-

ville) B.: A Reference Grammar of Modern Turkish (H. Nowka)

Taeschner, F., s. Turkologie

Teeuw, A., and H. W. Emanuels: A Critical Survey of Studies on Malay and Bahasa Indonesia (H. Mohring).

Temir, A., s. Turkologie

Thierry, G. J. $\dagger$, s. Studies on Psalms

Tietze, A.: Turkish Literary Reader (J. Németh)

Till, W. C.: Das Evangelium nach Philippos hrsg. u. übers. (J. Leipoldt $t$ )
Spalte 179 502 505 391 487 487 373
Tomson, E.: Die Volksrepublik China und das Recht nationaler Minderheiten (M. H. van der Valk)

Toulmin, R., s. Lamotte, E.

Tsien, T.-H.: Written on Bamboo and Silk (A. Bul. ling). . . . . . . . . . . . . . . . . .

Turkologie. Mit Beiträgen von A. v. Gabain, O. Pritsak, N. Poppe, J. Benzing, K. H. Menges, A. Temir, Z. V. Togan, F. Taeschner, O. Spies, A. Caferoglu, A. Battal-Taymas $=$ Handbuch der Orientalistik, hrsg. v. B. Spuler, I. Abt. V, 1 : Altaistik. I. (J. Német h) .

Turner, R. L.: A Comparative Dictionary of the Indo-Aryan Languages. III u. IV (W. Rau)

Vandier d'Abbadie, J.: Nestor L'Hôte (1804-1842) (A. Hermann)

- Manuel d'Archéologie Égyptienne. IV. (G.

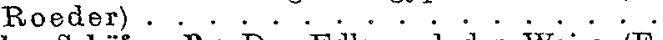

Weber-Schäfer, P.: Der Edle und der Weise (F. Tókei)

Weisweiler, M.: Von Kalifen, Spaßmachern und klugen Haremsdamen (S. Simon) . . . . . .

Williams, R.: Jaina Yoga (F. J.Meier)

Wiseman, D. J.: Catalogue of the Western Asiatic Seals in the British Museum. I. (R. M. Boeh -

Woolley, L.: Ur Excavations. IX: The Neo-Babylonian and Persian Periods. With a Contribution by M. E. L. Mallowan (A. Moortgat).

Woude, A. S. van der, s. Studies on Psalms

Yadin, Y.: The Finds from the Bar Kokhba Period in the Cave of Letters (O. EiB feldt).

Yasuda, K.: ,The Japanese Haiku“" (L. Gromkovskaya)

Zajaczkowski, A.: Poemat irański Husrev-u-Sirin w wersji osmańsko-tureckiej Seyhî (J Rypka) .

Ziadeh, F. J., s. Mahmassani, S.

Ziegler, K.-H.: Die Beziehungen zwischen Rom und dem Partherreich (J. Wolski) . . . .

\section{Verzeichnis der Mitarbeiter}

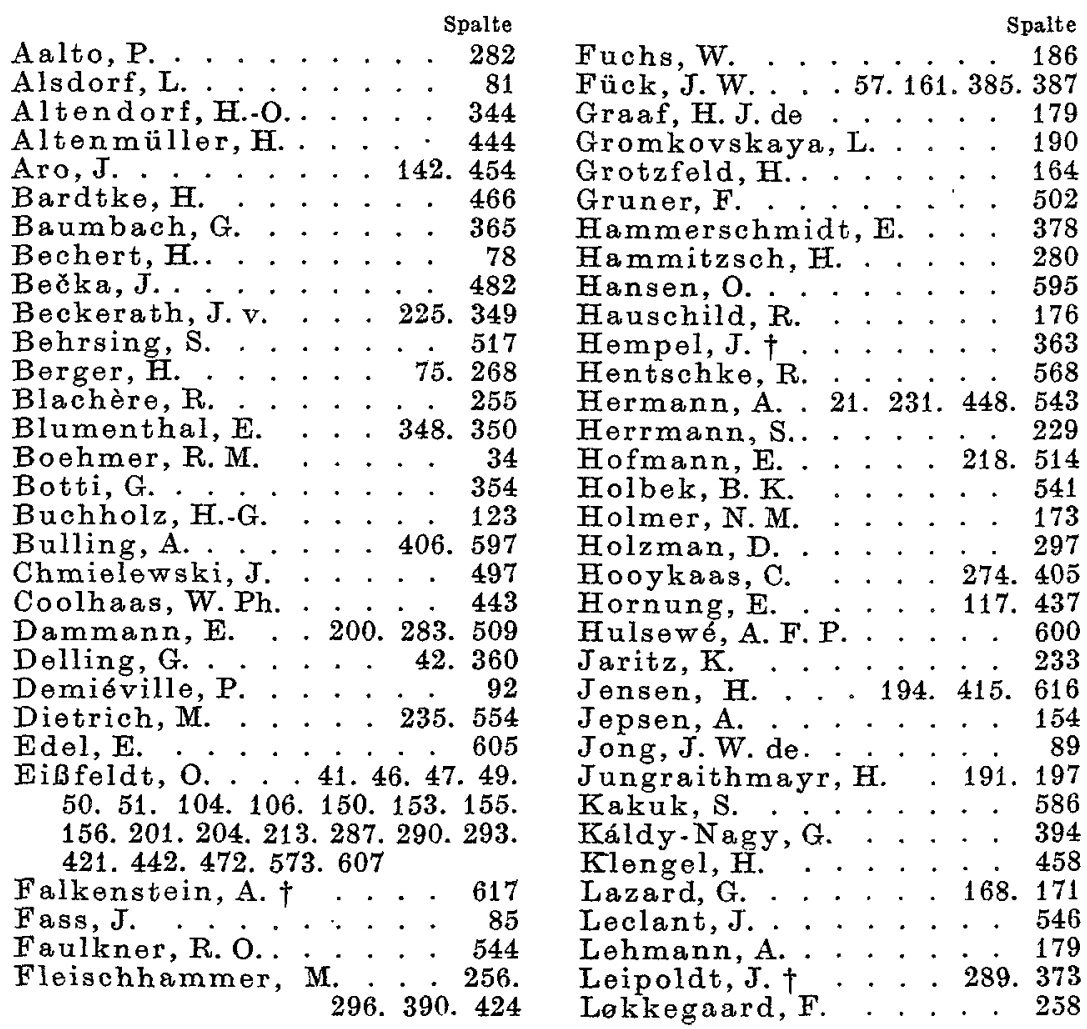

Loretz, O. 235. 554

Markov, W. ...... 218

Meier,F.J. . . . . . . . 269

Mellink, M. J. . . . . 39. 145

Menges, K. H. . 63

Mensching, G. . . 295. 297.422

Meyer, R. . . 52. 253. 374. 412 467. 510.576

Mode, H. . . . . . . 400

Mohring, H. . . . . . 83

Moorgat, A. . . . . . . . 140

Morenz, S. . . . . . . 202

Müller, D. . . . . . 135

Müller, W. W. . . . 54. 254. 603

Nage1, P.. . . . . 5 289. 416

Nagel,W. . . . . . . . 166

Németh, J. . . . . 165. 479

Nowka, H. . . . . . . . . 392

Oelsner, J. . . . . . . . . $\mathbf{5 8 0}$

OBwald, E. . . 48.151.246.462.

565. 573

Otten, H. . . . . . . . . 614

Paret,R. . . . . . 411.602

Paul,E. . . . . . . 14. 219

Petráček, K. . . . 157

Pinnow, H.-J. . . . . . . 492

Pokora, T. . . . . . . . 296

Poucha, P. . . 84. 85. 188. 291 298. 425.518

Priese, K.H. . . . . . . . 315

Pulleyblank, E. G. . . . . 408

Ratchnevsky, P. . . . 182

Rau, W. ... 204. 286. 490. 592

Reychman, J. . . . . . 396 


\begin{tabular}{|c|c|c|c|}
\hline Spalte & & Spalte & $T$ T \\
\hline ler, $\mathbf{K}$. & Schmithausen, L. & 74 & Valk, M. H. van der \\
\hline 132 & & 91.188 .505 & Vietze, H.P. . . \\
\hline 450 & & . . 460 & Vochala,J. \\
\hline •. * 325 & $\mathrm{n}, \mathrm{H}$. & 56 & Vorbichler, A. \\
\hline 99. 250. 370. 571 & Singer, H.-R. & 107 & Wallis, G. $\cdot 149.244 .371 .4$ \\
\hline . . . 487.590 & Skurzak, L. & $\therefore \quad 402$ & $\begin{array}{l}\text { Watt, W. M. } \\
\text { Wei } B, H . F\end{array}$ \\
\hline 251. 361. 613 & $\mathrm{n}, \mathrm{W}$. von & 356.419 .560 & WeiB, H.-F. $_{\text {W }}$ \\
\hline $\begin{array}{l}\text { Rypka, J. } \\
\text { Schenke, H.-M. }\end{array}$ & Spuler, B. . & . . 391. 474 & $\begin{array}{r}\text { Weller, F. . 95. } 177.272 .28 \\
417.601 .60\end{array}$ \\
\hline$\therefore$ 164.387. 404 & Tau & $\begin{array}{r}208 \\
89\end{array}$ & $\begin{array}{r}417.601 .6 \\
. \quad \cdot 136.5\end{array}$ \\
\hline . . . . . 265 & & 411 & $\therefore . .100$ \\
\hline 44 & öpfer, B. & 477 & obel, \\
\hline
\end{tabular}

Zeitschriftenschau: Acta Orientalia 602-African Studies 94. 200 - Andrews University Seminary Studies 201 Annales du Service des Antiquités de l'Egypte $202-$ Annals of the Bhandarkar Oriental Research Institute 204. 286 - Archiv Orientální 603 - Berytus. Archeological Studies 204. 287 - Bibliotheca Orientalis 605 Biblische Zeitschrift 607 - Bulletin of the American Schools of Oriental Research 510 - Bulletin of the Faculty of Arts 411 - Bulletin de la Société de Linguistique de Paris 514 - East and West 95.609Hamizrah Hehadash 99 - Harvard Journal of Asiatic Studies 287 - History of Religions 613 - Journal Asiatique 517 - Journal of Cuneiform Studies $614-$ Journal of Near Eastern Studies 104 - Kirjath Sepher 412 - Language 415. 616 - Le Muséon 289.
416 - Oriens Antiquus 106 - Oriens Extremus 417 Orientalia 419 - Palestine Exploration Quarterly 290. 421 - Przeglad orientalistyczny 291 - Revue Biblique 293 - Revue de l'Histoire des Religions 295. 422 Rocznik orientalistyczny 296 - Studies in Bibliography and Booklore 296. 424 - Studi e Materiali di Storia delle Religioni 297 - Sumer 617 - Töhōgaku 297 - Trudy dvadcat' pjatogo Meždunarodnogo kongressa vostokovedov 298.425. 518 - Wiener Zeitschrift für die Kunde des Morgenlandes 107 - Zeitschrift für Ägyptische Sprache und Altertumskunde 315 -

Zur Besprechung eingegangene Bücher siehe 2. und 3. Umschlagseite jeder Nummer 
